\title{
L'opéra idéal selon la Lettre à Meyerbeer
}

Rêverie critique et utopie musicale

\section{Olivier Bara}

\section{(2) OpenEdition}

\section{Journals}

Édition électronique

URL : http://journals.openedition.org/recherchestravaux/234

DOI : 10.4000/recherchestravaux.234

ISSN : 1969-6434

Éditeur

UGA Éditions/Université Grenoble Alpes

Édition imprimée

Date de publication : 15 avril 2007

Pagination : 141-154

ISBN : 978-2-84310-107-7

ISSN : 0151-1874

Référence électronique

Olivier Bara, "L'opéra idéal selon la Lettre à Meyerbeer », Recherches \& Travaux [En ligne], 70 | 2007, mis en ligne le 04 mars 2009, consulté le 08 septembre 2020. URL : http://journals.openedition.org/ recherchestravaux/234; DOI : https://doi.org/10.4000/recherchestravaux.234 
Olivier BARA

Université Lumière-Lyon 2, UMR LIRE

\section{L'opéra idéal selon la Lettre à Meyerbeer Rêverie critique et utopie musicale}

La lettre XI, "À Giacomo Meyerbeer », numérotée VIII lors de sa parution dans la Revue des Deux Mondes ${ }^{\mathrm{I}}$, est le point d'aboutissement d'un fil thématique courant à travers les Lettres d'un voyageur. Ce fil, entrelacé à d'autres fils, tisse une trame textuelle susceptible d'atténuer l'impression de disparité laissée par le recueil. Dans la lettre précédente "tonnait " l’orgue de Fribourg face au démiurge impassible, Mooser, l'homme qui commandait aux orages. Dans la lettre VII, "À Franz Liszt ", résonnait la puissante harmonie de la " république " symphonique beethovénienne. La lettre VI, "À Éverard ", opposait au républicain fanatique, «Vandale » irrespectueux de l'art, la figure du "véritable artiste ", Berlioz, appelé lui aussi à agir et à créer dans la « jeune Jérusalem ", " la cité nouvelle de l'intelligence " (p. 8IO ; p. I9I I²). Les premières lettres, vénitiennes, bruissaient quant à elles de romances et de barcarolles, chansons des rues et des canaux, chœurs de gondoliers et de facchini. Dans la lettre I, enfin, souvenirs tyroliens et expériences musicales s'appelaient, se reflétaient, se confondaient dans le double miroir des sens et de la mémoire.

Ce n'est pas dans la course sinueuse de ce fil musical que réside l'originalité de ces Lettres de Sand : bien des récits de voyage convoquent l'univers des

I. La lettre numérotée VIII (future lettre XI du recueil) comme la lettre numérotée VII (future lettre X) paraissent dans le numéro du I5 novembre 1836.

2. Selon la convention que nous adoptons pour ce volume: G. Sand, Lettres d'un voyageur, pages de l'édition de G. Lubin puis pages de l'édition de H. Bonnet. 
sons au côté de l'empire des images pour précipiter, dans l'écriture de la marche, quelque expérience synesthésique 3 . Plus spécifiquement, les Lettres d'un voyageur privilégient trois questions musicales récurrentes. La première touche au sens de la musique, à sa puissance figurative transmuant l'expérience auditive en voyage immobile, par la grâce confondue des modulations sonores et des visions imaginaires. La seconde question concerne cet artiste majuscule qu'est le musicien, dont la figure idéale se décline en trois noms, destinataires directs ou indirects des lettres : Liszt, Meyerbeer, Berlioz. La troisième question musicale accentue la tension entre concert et théâtre lyrique, musique avec ou sans représentation spectaculaire : la Symphonie pastorale de Beethoven revient comme un leitmotiv, accordant la suprématie aux concerts du Conservatoire au détriment des prestiges de l'Opéra ; à Venise, d'ailleurs, l'opéra sort de l'Opéra, les airs passent des gosiers des divas et divi à ceux des dilettanti ignorants de la musique écrite, capables de purger les cavatines et les arias de leurs factices ornements de théâtre.

La lettre XI est le point de convergence de ces trois questions comme si elle constituait un aboutissement de la thématique musicale dans les Lettres d'un voyageur. Tel est du moins l'effet créé par la constitution du recueil ${ }^{4}$. Les trois noms de Meyerbeer, Liszt et Berlioz s'y trouvent cités comme y est rappelée la haute mission de l'artiste, pédagogue et guide. Le Voyageur s'y concentre sur le spectacle lyrique, à travers Les Huguenots de Meyerbeer, en un éloge paradoxal du spectacle lyrique qui s'apparenterait plutôt - on le verra - à une destruction du grand opéra historique, de ses rites sociaux, de ses artifices vocaux comme de ses prestiges scéniques. Cette condamnation des signes dramatiques et scénographiques jugés redondants est portée au nom de l'éloquence musicale naturelle et de la faculté d'imagination de l'auditeur - où l'on retrouve l'attachement au pouvoir figuratif de la musique. Ainsi, en un vaste finale engendré par le geste même de recollection des lettres, la onzième expose dans leur plénitude les trois thèmes musicaux esquissés, préparés, variés dans les parties antérieures.

Mais cette lettre révèle aussi son étrangeté. Elle serait d'abord la lettre où la musique surgit le moins "naturellement ». La présence de cet adverbe sous la plume de Sand, au début du treizième paragraphe, est peut-être une cheville du discours trop voyante pour ne pas être rhétorique : «Naturellement, ces chants imaginaires prirent dans mon cerveau la forme du beau cantique de l'opéra des Huguenots [...]» (p. 92I ; p. 298). Là où les autres lettres culti-

3. Voir B. Didier, "Voyages et opéra, Lettres d'un voyageur ", George Sand écrivain, "Un grand fleuve d'Amérique ", Paris, PUF "Écrivains ", 1998, p. 29I-310.

4. Sur cet " effet recueil ", voir l'article de Marie-Ève Thérenthy dans le présent volume. 
vaient les échanges fluides et réciproques entre lieux visités, souvenirs ou auditions musicales, cette lettre XI donnerait la version presque caricaturale de tels fondus enchaînés en faisant surgir le spectacle des Huguenots à l'Opéra de Paris de la visite de l'église réformée de Genève, temple hanté par les voix du martyre calviniste. De plus, à l'association récurrente chez le Voyageur solitaire entre la musique et le paysage, les montagnes du Tyrol, l'eau des canaux vénitiens ou l'orage de Fribourg, la lettre XI ferait succéder l'ancrage tout urbain de l'opéra, spectacle collectif, venant rompre par ses fastes et ses artifices l'échange dialectique préservé jusque-là par les lettres entre la nature et la cultures. Pour la première fois, en outre, une lettre accueillerait un texte étranger au cadre épistolaire, puisque celle-ci ressortit davantage, en sa partie centrale, à la critique musicale ou au feuilleton dramatique qu'au récit des errances physiques, imaginaires et intellectuelles. Enfin, la lettre à Meyerbeer est traversée par une question propre, lancinante, celle de la légitimité : légitimité du voyageur écrivain qui ose disserter sur la musique et sur l'art lyrique. La question est largement mise en scène à travers les masques de l'énonciation : celui qui dit " je " est un "pauvre poète ", un " écolier sans conséquence» (p. 917 ; p. 294), un « ignorant» (p. $928 ;$ p. 305) qui a « la voix fausse et ne sai $[t]$ jouer d'aucun instrument" (p. $930 ;$ p. 306). Cette posture "auctoriale " n'a rien d'exceptionnel, et elle est aussi adoptée dans les lettres privées de Sand, à Liszt par exemple, le I5 mai 1836 :

La passion de la musique, à laquelle je n'entends rien, [...] me jette dans des extases et dans des ravissements qui ne sont pas de ce monde, et [...] me fait accoucher de temps en temps de quelque déclamation bien ampoulée et bien bête, après quoi je m'imagine avoir fait merveille et j'ai l'estomac tout à fait soulagé 6

Dans les Lettres d'un voyageur, cette tonalité énonciative confere à la lettre à Meyerbeer son originalité ; elle crée une dissonance susceptible de rompre l'harmonie du recueil.

S'agit-il d'une posture destinée à conjurer le soupçon d'imposture ? N'estce pas plutôt une stratégie délibérée, consistant à adopter le point de vue du naïf, de l'homme imprévisible et ouvert, autre nom de ce Voyageur libre de préjugés, réceptif et disponible aux mille sollicitations du monde? Tout dans la lettre, de la position énonciative à la structure thématique, est faussement naturel et délibérément construit : le texte trouve son accomplissement dans

5. Voir l'article de Ph. Berthier, "Lettres d'un voyageur: le cru et le cuit ", Ville, campagne et nature dans l'œuvre de George Sand, S. Bernard-Griffiths (dir.), Clermont-Ferrand, Presses universitaires Blaise-Pascal «Révolutions et Romantismes ", 2002, p. 135-I47.

6. G. Sand, Correspondance, op. cit., vol. III, p. 37I. 
cette fiction devenue illusion de vérité, dans cette énonciation savamment mise en scène jusqu'à se faire passer pour voix de la nature. Autrement dit, dans cette lettre centrée sur une utopie opératique, projet de réforme du grand opéra, art de la convention, le projet esthétique sandien de célébration des noces de la culture et de la nature, de l'illusion et de la vérité, apparaît dans toute sa cohérence. Loin de n'être qu'un " caprice épistolaire ${ }^{7}$ ", la lettre XI, dans son excentricité même, peut révéler sa densité organique et trouver finalement une place légitime dans le volume des Lettres d'un voyageur.

La Lettre à Meyerbeer naît d'un spectaculaire décentrement géographique du discours : cela consiste tout simplement à signer de Genève une lettre consacrée à l'Opéra de Paris. Ordinairement, le lieu commun du récit de voyage, en matière musicale, consiste à parler depuis telle ville, de préférence italienne, des mœurs lyriques locales, de la sociologie des spectacles, de leur esthétique, beautés et ridicules mêlés, selon un jeu de comparaisons avec la ville d'origine du voyageur-témoin, touriste, esthète et moraliste. Liszt, dans ses Lettres d'un bachelier ès musique publiées dans la Revue et Gazette musicale sacrifie à ce topos du voyage en Italie, en offrant aux lecteurs son point de vue sur l'opéra à Milan". Dans sa Lettre d'un voyageur destinée " à M. George Sand » et publiée dans la même Gazette musicale le 6 décembre I835, Liszt propose à son destinataire direct, George Sand, et à ses destinataires indirects, les lecteurs de la gazette, une " chronique musicale de Genève, la Rome protestante9 ". La Lettre de Liszt est logiquement datée : "Genève, 23 novembre. " Sand s'inspire visiblement de ce texte qu'elle réécrit et transforme en sa lettre XI, datée aussi de Genève et ouverte par une description des "murailles dénudées » de l' " église dépouillée ${ }^{\mathrm{ro}}$ » déjà évoquées par Liszt. Mais cet ancrage fictif de l'énonciation - Genève, le temple - n'entraîne pas, comme chez Liszt, le discours ironique sur la pratique musicale des protestants, seul art rescapé de l'exclusion hors du temple de la peinture et de l'architecture. Liszt évoquait satiriquement une chorale " dont une bonne

7. Th. Marix-Spire, Les Romantiques et la musique. Le cas George Sand, I804-I838, Paris, Nouvelles Éditions latines, 1954, p. 5 IO.

8. F. Liszt, Lettres d'un bachelier ès musique, $\mathrm{n}^{\circ} \mathrm{IV}$ et VI, Revue et Gazette musicale, 25 mars et 27 mai I838. Reprises dans Pages romantiques, édition de J. Chantavoine, Paris, Félix Alcan, 1912. Ces lettres de Liszt dédiées "à un poète voyageur " sont conçues comme le prolongement des Lettres d'un voyageur de Sand.

9. F. Liszt, Lettre d'un voyageur, in Pages romantiques, op. cit., p. 87. Cette lettre est la réponse du compositeur et ami à la lettre VII "À Franz Liszt " publiée par Sand dans la Revue des Deux Mondes le $\mathrm{I}^{\mathrm{er}}$ septembre I835.

Io. Ibid., p. 88. 
moitié protestait avec un zèle si fanatique contre la mesure et l'intonation ${ }^{\mathrm{II}}$ ». Sand, quant à elle, glisse sur les cantiques entendus après le prêche, pour cultiver l'illusion auditive des invocations religieuses, "sous toutes les formes " (p. 921 ; p. 298), papistes ou huguenotes. Surtout, ces pages liminaires de la missive genevoise, calquant la Lettre d'un voyageur de Liszt pour mieux s'en détacher, préparent l'émergence du discours central consacré à l'opéra Les Huguenots de Meyerbeer - le destinataire direct de la lettre. L'illusion auditive créée par l'austérité des lieux se poursuit fictivement par le souvenir musical et visuel du dernier acte de l'opéra, l'acte de la Saint-Barthélemy :

[...] et tandis que je croyais entendre au-dehors les cris furieux et la fusillade serrée des catholiques, une grande figure passa devant mes yeux, une des plus grandes figures dramatiques, une des plus belles personnifications de l'idée religieuse qui aient été produites par les arts dans ce temps-ci, le Marcel de Meyerbeer. (loc. cit.)

Tel est le mirage, telle est la transition, menant de la description du temple genevois au vif de la lettre : l'évocation du grand opéra.

Cet enchaînement entre la visite du temple genevois et l'analyse des Huguenots relève moins du procédé artificiel que de la stratégie critique. Au lieu d'adopter le point de vue du spectateur parisien, confronté depuis sa place à l'Opéra aux signes dramatiques, jugeant de la vérité théâtrale par l'illusion éprouvée, Sand part de la réalité du culte protestant, se fonde sur le signifié, constaté de visu, pour remonter aux signes censés le représenter - le grand opéra de Meyerbeer. Le point de vue choisi dans le dispositif épistolaire est ainsi celui de l'histoire, point à partir duquel le destinateur peut adresser au destinataire éloges et blâmes : éloge de la vérité dramatique atteinte avec cette figure de huguenot, Marcel, "vraie comme l'histoire " (loc. cit.) ; blâme en revanche pour Scribe, ses "versiculets insignifiants " et ses pauvres « accessoires» (p. 927 ; p. 303). Le plus grand compliment que le critique-voyageur puisse faire à Meyerbeer est d'affirmer non pas que son opéra appelait la vision d'authentiques lieux protestants, plus ou moins bien reproduits sur scène, mais que la visite d'un temple gagnait en intensité au souvenir des plus belles pages de son opéra. L'art doit nourrir l'expérience du réel et non dupliquer le monde visible - idée qui contribue déjà à rejeter hors de la vue les fastes encombrants, redondants et décevants, de la représentation lyrique. L'œuvre musicale ne remplace aucunement l'expérience mais l'enrichit, confere à l'instant de la perception sa profondeur et ses reliefs, l'enveloppe de toutes les harmoniques des souvenirs personnels, historiques

II. Ibid., p. 89. 
et artistiques. Ainsi, fiction et vérité, apparences et réalité se fécondent mutuellement dans cet au-delà de toute reproduction qu'est l'œuvre accomplie.

Le décentrement géographique se double, dans la lettre XI, d'un décalage temporel également signifiant. La Lettre à Meyerbeer est datée de septembre I836, ce qui fait coïncider son écriture supposée avec l'époque du voyage de George Sand à Genève, où elle rejoint Liszt et Marie d'Agoult, du 4 septembre au $\mathrm{I}^{\mathrm{er}}$ octobre. L'audition par Sand d'un prêche calviniste est ainsi attestée par Georges Lubin, à la date du I8 septembre ${ }^{\mathrm{r} 2}$. Tout s'ajusterait parfaitement sur le double plan de la biographie et de la référentialité. Pourtant, deux étrangetés subsistent. La première, la plus visible, est le décalage flagrant entre la date fictive de la lettre, septembre, la date de publication dans la Revue des Deux Mondes, Is novembre, et la date de création des Huguenots, 29 février 1836, soit huit mois et demi avant la parution de la Lettre à Meyerbeer, compte rendu à contretemps d'un événement lyrique retentis$\operatorname{sant}^{13}$. Le second flottement masqué par le " cachet " de la lettre concerne l'ancienneté du projet sandien d'écrire sur Les Huguenots : dès le I4 avril, dans l'effervescence parisienne de la création de l'opéra de Meyerbeer, Sand demande à Buloz d'intercéder auprès du compositeur afin d'obtenir une loge : «Il est indispensable que j'entende cela encore une fois avant d'en parler $[\ldots]^{14}$. » Un mois plus tard, le I5 mai, Sand écrit à Liszt depuis La Châtre : "J'ai été à Paris passer un mois, j’y ai vu tous nos amis : Meyerbeer, sur qui j'écris assez longuement à l'heure qu'il est (j'adore Les Huguenots) ${ }^{15}$. " Malgré une volonté première de réagir "à chaud " aux représentations du nouvel opéra, le projet a été différé, momentanément abandonné - repris plus tard et ailleurs.

Ce retard s'explique par l'évidente hésitation de l'auteur à aborder un sujet musical ailleurs que dans le cadre de la fiction, à traiter pour la première fois dans la presse d'une œuvre lyrique, commentée et jugée par cet " écolier sans conséquence " mis en scène dans la lettre XI. Cette manière de différer l'achèvement et la publication de son texte permet aussi à Sand de mettre à profit le décalage temporel, interdit au feuilletoniste, de refuser l'urgence qui est la loi du critique professionnel. La Lettre à Meyerbeer échappe de la sorte aux tares dénoncées en ses derniers paragraphes, justement consacrés à la cri-

I2. G. Sand, Correspondance, op. cit., vol. III, p. 552.

I3. Voir M.-H. Coudroy, Les Créations de Robert le Diable et des Huguenots de Meyerbeer face à la critique, Paris, CNSM, 1979.

I4. G. Sand, Correspondance, op. cit., vol. III, p. 339.

15. Ibid., p. 370. 
tique : elle ne sera pas mise au rang des « trente ou quarante jugements littéraires qui s'impriment le lendemain de l'apparition d'une bluette quelconque " (p. $932 ;$ p. 308). Le temps creusé entre la découverte du nouvel opéra et son évocation dans la lettre est aussi celui de l'expérience et de la vie, sans lesquelles l'émotion artistique s'exténue, tuée par la frénésie des stimulations comme par l'obligation de tout juger tout de suite. Articulation entre l'émotion vive et la jouissance artistique, ressourcement de la faculté d'émotion dans l'expérience du voyage : jusque dans sa digression finale sur la critique, la lettre XI conserve la cohérence de son projet esthétique et éthique.

Ainsi, la lettre de Sand peut s'apparenter à quelque critique en liberté, dégagée de l'événement, de la tâche ingrate de résumer et de disséquer sur le vif le nouvel ouvrage. Elle mise volontiers sur la distance temporelle pour excuser les erreurs - par exemple la confusion malheureuse entre l'acte III et l'acte IV pour la scène entre Valentine et Raoul, ou la réduction abusive du personnage du comte de Nevers à l'état de simple comparse qui a " quatre paroles à dire dans le libretto " (p. 927 ; p. 304). Échappant à la catégorie du feuilleton musical, le texte de Sand passe volontiers sous silence certains éléments fondamentaux de l'art du grand opéra et de Meyerbeer : on ne lira rien ou presque sur l'instrumentation du compositeur ; on trouvera une analyse dramaturgique bien limitée, réduite au rejet « de la toile peinte en rouge de l'Opéra et de l'escamotage adroit de six quinquets » (p. 924 ; p. 30I), ou à la critique sans nuance et sans grande perspicacité du livret de Scribe ${ }^{16}$. Quant à la scène de Raoul et Valentine, un des sommets de l'œuvre et un des plus puissants duos de l'histoire de l'opéra, Sand se contente de s'exclamer à son propos : «Quel duo!»- ce qui est un peu mince ${ }^{17}$. Le Voyageur semble passer à côté des véritables enjeux dramatiques et musicaux du grand opéra historique en cinq actes né à la fin de la Restauration avec La Muette de Portici de Scribe et Auber (I828) : la convergence du son et de l'image, la gradation des effets et la superposition des masses musicales, l'unité dramatique trouvée au point de jonction entre l'intrigue privée et la mise en scène d'une rupture historique $^{\mathrm{r}}$. Seules ses remarques laudatives sur la vérité dramatique du der-

I6. La critique parisienne ne fut pas plus perspicace sur la réussite du travail de Scribe (l'articulation entre une intrigue passionnelle et un drame collectif) : on discuta surtout des inexactitudes historiques et de la part jugée trop belle faite aux protestants. Voir M.-H. Coudroy, Les Créations de Robert le Diable et des Huguenots de Meyerbeer face à la critique, op. cit., passim.

17. Sand concède toutefois que la scène est " très pathétique, très lugubre, très effrayante, et nullement anacréontique ", comme l'ont dit certains critiques (p. 927 ; p. 304).

I8. Les Huguenots est une des grandes (et rares) réussites du grand opéra dans son ambition de donner une représentation globale de la réalité sociale, susceptible de faire graviter tous les 
nier trio, scène " coupée et brisée, parce que la situation l'exige ", " musique de passion vraie et d'action vraisemblable» (p. 927-928; p. 304), rendent sincèrement hommage à l'intelligence théâtrale de Meyerbeer. Mais la visée de cette lettre XI est tout autre que la critique d'art savante. Elle cultive à rebours de tout "professionnalisme " la subjectivité du témoignage et du jugement, comme la feinte nonchalance de la digression; elle s'attache davantage à mettre en scène une voix et un discours originaux, isolés dans le concert parisien des voix et des discours.

Une dernière caractéristique renforce ainsi la dimension intempestive de la lettre XI : à l'art du contretemps succède celui du contrepoint critique. La Lettre à Meyerbeer est certes la partie d'un tout appelé Lettres d'un voyageur, élaboré en recueil à partir de février I837. Mais la lettre s’insère aussi, au moment de sa composition et de sa réception immédiate, dans un corpus de textes beaucoup plus large comprenant entre autres les discours critiques abondamment suscités par l'événement parisien de la création des Huguenots. En particulier, Sand noue dans la lettre XI un dialogue polémique avec Henri Blaze de Bury, en quelque sorte son rival immédiat en fait de critique puisque ce dernier a publié, le I5 mars I836, dans la même Revue des Deux Mondes, un long article consacré généralement aux "poètes et musiciens de l'Allemagne " et particulièrement à « M. Meyerbeer ». Le dialogue à distance Sand-Blaze de Bury est du reste attesté par la réponse privée envoyée par Meyerbeer à l'auteur de la lettre à lui adressée : l'auteur des Huguenots demandait à Sand de supprimer les phrases critiquant directement ce « jeune auteur " avec qui il avait fait désormais la paix ${ }^{19}$. Si le nom de Blaze de Bury est effacé, remplacé par le pronom indéfini " on " ("On a dit à propos des Huguenots [...]»; p. 923 ; p. 300), l'ombre de ses jugements pèse sur le texte sandien enclin à s'en démarquer régulièrement.

Certes, une convergence s'opère dans la condamnation commune du livret de Scribe et de ses «figures mesquines » selon Blaze de Bury; mais ce dernier ne rêve pas à l'instar de Sand d'écrire lui-même un livret : il attend seulement l'ère du compositeur-librettiste pouvant "se suffire à lui-même ${ }^{20}$ ". Ailleurs, Sand n'a de cesse de se poser en s'opposant. Lorsque Blaze de Bury affirme qu'il n'existe pas de musique catholique ni de musique luthérienne,

éléments secondaires du spectacle autour d'une unité centrale. Voir l'article de G. de Van, « Le grand opéra entre tragédie lyrique et drame romantique ", Il Saggiatore musicale, ${ }^{\circ}$ 2, I996, p. 325-360.

I9. D'après Georges Lubin dans son édition des Lettres d'un voyageur, " Notes et variantes ", note $\mathrm{n}^{\circ}$ 3, p. 1496.

20. Revue des Deux Mondes, I5 mars I836, p. 694-695. 
que tout est affaire de sentiment dans l'interprétation et non d'harmonie ou de mesure ${ }^{21}$, Sand répond par une défense vibrante du sens et de la couleur propres de la musique, indépendamment de ses conditions extérieures d'exécution :

[...] comme si la musique n'était qu'un habile arrangement de sons plus ou moins bien combinés pour flatter l'oreille, et que le rythme seul approprié à la situation dramatique suffit pour exprimer les sentiments et les passions d'un drame lyrique ! J'avoue que je ne comprends pas, et je me demande si la principale beauté de Guillaume Tell ne consiste pas dans le caractère pastoral helvétique, si admirablement senti et si noblement idéalisé. (P. 923 ; p. 300.)

Un autre emportement, moins vif, touche à la question morale lorsqu'elle évoque cette scène de l'acte III - en réalité acte IV - « que des puritanismes, d'ailleurs estimables, ont incriminée un peu légèrement" (p. 927 ; p. 304). Blaze de Bury dénonçait en effet les " misérables scènes d'alcôve ${ }^{22}$ " indignes de l'opéra. Et lorsque le même critique regrette l'abus des modulations au détriment de la pureté mélodique chez Meyerbeer ${ }^{23}$, Sand affirme qu'il « n'est pas besoin d'une mélodie complète ", et qu'une seule modulation suffit à passer de l'ombre à la lumière - comme cette "modulation d'Alice au pied de la croix» (p. 925; p. 302) dans Robert le Diable, qu'elle dit se faire jouer en ses moments de spleen. Enfin, face aux sarcasmes du jeune critique sur l'église protestante («cette femme stérile qui [...] n’a jamais pu tirer une seule goutte de lait de ses mamelles de pierre $\left.{ }^{24} »\right)$, Sand s'en prend à ces « hommes d'actualité " qui affirment que " la réforme avait été, dès sa naissance, la plus plate idée du monde, et la forme religieuse de cette idée la plus pauvre et la plus aride de toutes les formes" (p. 918; p. 295). Une nouvelle fois, ici dans la défense du protestantisme, la réponse différée de Sand se fait presque terme à terme. On pourrait multiplier les exemples pour souligner comment cette lettre, née de l'échange et de la polémique, est tissée de voix étrangères auxquelles répond la voix du signataire. Il s'agit, sur la scène médiatique, d'imposer un ton et un nom, et cela sur un terrain, la critique musicale, où on ne l'attendait guère.

Un semblable dialogue unit la lettre XI aux textes de Liszt publiés dans la Revue et Gazette musicale : l'échange amical, dialogue privé prolongé sur la

2I. « Je pense que M. Meyerbeer, en écrivant sa partition s'est un peu trop préoccupé de la question religieuse ; il a voulu faire de la musique luthérienne [...] ; comme s'il y avait aujourd'hui une musique catholique, une musique luthérienne ", ibid., p. 684.

22. Ibid., p. 703 .

23. "En outre, M. Meyerbeer abuse étrangement de plusieurs effets, par exemple de ceux produits par la modulation ", ibid., p. 690.

24. Ibid., p. 689. 
scène publique, remplace ici la discussion polémique. De la Revue des Deux Mondes à la Gazette musicale vont et viennent les lettres et les hommages, les dédicaces et les réponses. Un réseau textuel enserre ainsi les « Considérations sur la place des artistes » de Liszt, du 3 mai au I5 novembre 1835, les lettres sandiennes, la Lettre d'un voyageur à $M$. George Sand, de décembre I835, puis les lettres lisztiennes "d'un bachelier ès musique ", de février I837 à mars I839. Pour ne prendre qu'un exemple du jeu à distance et en public pratiqué par les deux complices et amis, Liszt reprend, dans son article du 26 juillet I835, l'expression "homme de génie " appliquée à $B_{e r l i o z}^{25}$ : en note, il précise qu'une " femme célèbre, G. Sand ", l'a utilisée dans un article de la Revue des Deux Mondes - il s'agit en effet de la lettre «À Éverard ». En conclusion de la Lettre XI, Sand revient sur Berlioz, modèle de l'artiste incompris selon Liszt, pour renouveler son propre hommage au "grand artiste méconnu» (p. 935 ; p. 3II). Mais une nouvelle fois, la proximité des thèmes abordés ne doit pas offusquer les différences que Sand se plaît à faire entendre dans ses propres lettres. Ainsi du thème de la critique, abordé avant Sand par Liszt, le 30 août I835. Si la métaphore de l'" eunuque » est commune aux deux textes ${ }^{26}$, comme l'articulation établie entre critique et enseignement, les conclusions sont divergentes : l'un, Liszt, se prononce pour les examens préparatoires aux fonctions de critique ${ }^{27}$; l'autre, Sand, prône la rareté d'une critique dégagée de l'asservissement au joug de l'opinion ${ }^{28}$. Loin de s'abandonner à une parole solitaire, le Voyageur fictif des lettres de Sand se nourrit de la parole des autres, y trouve l'élan d'une profération que la sociabilité ne condamne ni à l'éparpillement ni à la vacuité d'un simple écho.

Décentrement, contretemps et contrepoint, telles sont donc les conditions d'un discours autre sur le grand opéra. Ce triple décalage permet l'émergence d'une parole utopique, suffisamment détachée de la scène parisienne et

25. "Berlioz, homme de génie, homme populaire (et qui cependant restera toujours supérieur à sa popularité) ; Berlioz, l'artiste nouveau par excellence, le musicien du canon de juillet et de la France. " F. Liszt, "De la situation des artistes et de leur condition dans la société ", Pages romantiques, op. cit., p. 26.

26. Liszt écrit : "Le feuilleton se recrute plus habilement dans cette population d'incapacités spéciales, d'eunuques envieux ou oisifs [...]. " "De l'enseignement et de la critique ", Pages romantiques, op. cit., p. 59. Sand s'exclame: « [...] vous faisiez un métier d'eunuques et d'esclaves ; [...] soyez pères à votre tour " (p. $934 ;$ p. 310).

27. «Sans doute aussi que pour y remédier (en partie du moins), il serait nécessaire que nul ne pût s'arroger le droit de professer et d'exercer publiquement les fonctions de critique sans avoir passé un examen préparatoire et obtenu un diplôme. » F. Liszt, « De l'enseignement et de la critique ", Pages romantiques, op. cit., p. 60.

28. «[...] j'accorderais peu de moyens de gouverner l'opinion à ceux qui ont personnellement et exclusivement besoin de l'opinion» (p. 933 ; p. 310). 
des exigences du compte rendu critique pour épouser les formes de l'idéal. Cet idéal est frappé au sceau de la simplicité : Sand rêve à un opéra "simple comme l'antique" (p. 92I ; p. 298) que l'œuvre de Meyerbeer ne fait qu'approcher. Derrière la chaleur de l'éloge se cachent mal les reproches, car bien des formules peuvent relever de l'antiphrase ou révéler leur double tranchant comme cette déclaration : " [...] je vous confesse que je n'attendais pas une ouvre si intelligente et si forte"(p. 926 ; p. 302). Deux critiques majeures dominent : elles soulignent le poids de matérialité et de convention lestant l'opéra et l'empêchant de s'élever vers l'idéal. D'un côté, les décorations et les jeux de lumière paraissent mesquins et inutiles eu égard à la puissance figurative d'une musique se suffisant à elle-même. D'un autre côté, les codas, cadences et points d'orgue, ne sont que concessions au goût frelaté du " public grossier» et des " chanteurs inintelligents» (p. 929 ; p. 306), et transforment la scène en arène de cirque. A contrario, l'opéra idéal, esquissé en creux, est porté par une série d'exigences qui ont nom "vérité dramatique ", " passion vraie ", " action vraisemblable " (p. 928 ; p. 304). L'art musical, libéré des entraves de la convention, suppléerait aux carences techniques de la scénographie et à la naïveté des représentations scéniques ; il engendrerait par les seules ressources de l'harmonie les images mentales dans l'esprit ouvert et disponible de l'auditeur. Cet idéal, appelé " perfection possible» (p. 927 ; p. 303), Sand propose de l'atteindre en compagnie de Meyerbeer, dans le rêve d'une collaboration artistique qui engendrerait quelques Euménides ou une Mort d'Orphée. S'agirait-il d'une tragédie lyrique, d'une cantate ou d'un oratorio, ou encore de chœurs pour une tragédie à l'antique ? L'indistinction semble la condition d'émergence de cet idéal.

L’opéra rêvé par le Voyageur se découvre d'abord comme une destruction radicale du grand opéra historique à la française dont Guillaume Tell, Robert le Diable, La Juive, Les Huguenots, plus tard Le Prophète ou Dom Carlos sont les titres majeurs. Qu'une Lettre à Meyerbeer centrée sur Les Huguenots constate l'inutilité de la mise en scène et des décorations et prône le retour à l'antique constitue un formidable paradoxe : le grand opéra est essentiellement fondé sur l'exploitation dramatique de l'Histoire moderne, sur la convergence des fastes scéniques et de l'éloquence orchestrale, et sur l'intégration du bel cantisme dans la déclamation lyrique. Autrement dit, la lettre XI s'apparente à la destruction méthodique d'un genre voué à dominer la scène française, de la monarchie de Juillet au Second Empire.

Mais au profit de quel genre dramatico-lyrique cette destruction s'opèret-elle ? Et l'idéal de Sand relève-t-il vraiment de la projection utopique vers un ailleurs esthétique, inconnu et inouii ${ }^{29}$ ? En réalité, ce que propose la lettre dans le domaine du théâtre lyrique s'apparente à une reprise moderne de la 
réforme de Gluck. La simplification dramatique et musicale, condition d'accès au sublime, la préférence pour les sujets grecs, au détriment des sujets historiques romains des opéras métastasiens, constituaient déjà les recommandations du compositeur d'Alceste (1767). Le refus de voir le spectacle soumis à la seule exigence de virtuosité vocale, à travers la critique de l'aria da capo, apparaissait au XVIII siècle chez Gluck et son librettiste Calzabigi, mais aussi chez Marcello ou chez Porpora pour la musique religieuse ${ }^{30}$. Au début des années I830, l'inquiétude face à la prolifération des effets scéniques, la dénonciation du privilège accordé à la vue au détriment de l'écoute musicale sont des lieux communs de la critique. Liszt écrivait ainsi dans son article du 30 août I835: "L'Opéra s'éloigne de plus en plus de son but lyrique ; les machines, les décors, les costumes et le ballet tendent à absorber presque entièrement la musique $[\ldots]^{3 \mathrm{I}}$. " Sand s'inscrit ainsi dans le camp de tous ceux qui voient dans le grand opéra de la bourgeoisie triomphante une perpétuation moderne des fastes et des conventions baroques, dont les enchantements sont renouvelés par les progrès techniques et industriels de l'instrumentation, de l'éclairage et des machineries. Se trouve alors redoublée et légitimée la facticité des conventions sociales et mondaines : l'ornementation vocale n'est, selon Sand, que l'équivalent musical de la formule de politesse obligée au bas d'une missive. Ce sont autant de médiations dressant leurs écrans entre l'auditeur et la vérité musicale : l'ornementation n'est plus comprise, chez les romantiques, comme expression indirecte, métaphorique et idéalisée, des affects.

Une nouvelle fois, l'intérêt de la lettre XI réside moins dans son témoignage ou son invention critiques que dans sa cohérence esthétique, éthique et littéraire. On relèvera d'abord que le paragraphe final consacré à Berlioz donne symboliquement le dernier mot à l'auteur des Troyens, admirateur de Gluck, dont il remodèlera l'Orphée pour le confier à Pauline Viardot en I859.

29. Le texte de Sand serait-il tendu vers une forme synthétique à laquelle seul Wagner saura donner naissance? Chez Wagner, la musique absolue, donc autonome, n'existe pas : elle doit disposer d'un "motif formel ", le drame extériorisant dans les paroles, les gestes ou les décors ce qui demeure latent dans l'œuvre musicale. Le drame musical (Musikdrama) et l'œuvre d'art totale (Gesamtkunstwerk) transcendent le dualisme entre corps (mise en scène) et âme (œuvre) - dualisme sur lequel vient ici buter la pensée esthétique de Sand pour qui il s'agit de libérer «l'âme » de la musique des oripeaux de la scène. Voir C. Dahlhaus, Le Drame musical de Richard Wagner, Liège, Mardaga, I994.

30. Voir Querelle des Gluckistes et des Piccinistes, F. Lesure (dir.), Genève, Slatkine, 1984. Ce goût " pur » de Sand lui a été transmis par sa grand-mère : voir Histoire de ma vie, édition de G. Lubin, op. cit., vol. I, p. 626.

31. F. Liszt, "Des théâtres lyriques ", Pages romantiques, op. cit., p. 49, note $\mathrm{n}^{\circ} 2$. 
Plus que Meyerbeer, ce serait Berlioz «l'homme de l'avenir » (p. 929 ; p. 306) appelé par le Voyageur - du moins en I83632. Mais l'autre nom, lisible celuilà en filigrane dans la lettre, est celui du Philosophe de Genève : en une exclamation lyrique et satirique à la fois, Sand convoque implicitement le SaintPreux de la lettre sur l'opéra, dans La Nouvelle Héloïse : Ô toile ! ô carton! ô oripeaux ! ô machines!» (p. 924 ; p. 30I) déplore-t-elle ; "Le Ciel est représenté par certaines guenilles bleuâtres, suspendues à des bâtons ou à des cordes, comme l'étendage d'une blanchisseuse ", rapporte, avec plus de drôlerie, le héros de Rousseau ${ }^{33}$. Le constat de l'inutilité du décor, à qui est refusé le statut de signe dramatique, est le même. L'insistance récurrente, au long des Lettres d'un voyageur, sur le contenu visuel de la composition musicale fait aussi écho à l'article "Imitation" du Dictionnaire de musique où Rousseau écrit que la musique peut tout peindre et "semble mettre l'œil dans l'oreille ${ }^{34}$ ». L'ancrage genevois de la Lettre à Meyerbeer, ouverte dans un temple calviniste, contribue une nouvelle fois à tirer le discours vers la condamnation de la représentation et des images, dont l'opéra, art sensuel et profane, jouant avec les plaisirs de la mimesis, est le pourvoyeur impie. Ainsi, cette lettre « commencée dans une église » et « finie dans un théâtre » (p. 917 ; p. 294) est traversée par la tension entre sacré et profane. S’y exprime le désir de retremper les arts dans l'authenticité de la foi, de réformer la société et ses rites, notamment ces deux formes de sociabilité dévoyées que sont l'opéra et la critique, par la sainteté du lien fraternel ${ }^{35}$. Sur ce point, la lettre XI, influencée, via Liszt, par la pensée saint-simonienne, peut être lue comme le travail préparatoire du roman Consuelo.

L'opéra idéal selon George Sand demeure parfaitement imaginaire. Il n'existe que par les mots, seuls capables de creuser dans la fiction du voyage

32. Dix ans après la Lettre à Meyerbeer, Sand avouera à Delacroix s'être trompée en aimant Ingres et Berlioz: "Quand je pense que j'ai admiré, dans l'enfance de mon sentiment des arts, la première odalisque aux contours verts et au dos de sangsue blanche, et les symphonies en cuivre de Berlioz, je remercie le soleil du bon Dieu de m'avoir ouvert les yeux et les oreilles, car il faut être paralytique pour tomber dans de telles erreurs. " Correspondance, op. cit., vol. VII, p. 246.

33. J.-J. Rousseau, La Nouvelle Hélö̈se, seconde partie, lettre XXIII, in Euvres complètes, édition de B. Gagnebin et M. Raymond, Paris, Gallimard "Bibliothèque de la Pléiade ", vol. II, I964, p. 283.

34. J.-J. Rousseau, article "Imitation ", Dictionnaire de musique, cité par Th. Marix-Spire, Les Romantiques et la musique. Le cas George Sand, I804-I838, op. cit., p. 499.

35. «[...] la création d'une musique nouvelle est imminente, essentiellement religieuse, forte et agissante, cette musique qu'à défaut d'autre nom nous appellerons humanitaire résumera dans de colossales proportions le ThÉATRE et l'ÉGLISE. » F. Liszt, " De la situation des artistes et de leur condition dans la société ", Pages romantiques, op. cit., p. 66. 
cet espace improbable, capable de concilier la sociabilité et la solitude, l'expérience sensible et la vision intime, l'histoire et sa représentation, l'extériorité et l'intériorité, la nature et la culture, le profane et le sacré. Face à l'opéra, art menacé d'éclatement et de dispersion, en quête constante d'une totalité inaccessible, la lettre XI conjure l'angoisse de la déperdition et de la fragmentation : elle oppose au théâtre lyrique, vain spectacle des apparences, la communauté des concertistes, instrumentaux ou vocaux, comme la solidarité des critiques-créateurs, seuls capables d'approcher l'unité supérieure et l'austère vérité placées à l'horizon de l'écriture. 